\title{
Circulating zinc-a2-glycoprotein is reduced in women with polycystic ovary syndrome, but can be increased by exenatide or metformin treatment
}

\author{
Siyuan Zheng ${ }^{1)}$ *, En Liu ${ }^{1)}$, Ying Zhang ${ }^{1)}$, Tao Long ${ }^{1)}$, Xin Liu ${ }^{1)}$, Yi Gong'), Tingting Mai ${ }^{1)}$, \\ Huanling Shen ${ }^{1)}$, Hui Chen ${ }^{1)}$, Rong Lin ${ }^{1)}$, Yongxiong Zheng ${ }^{1)}$, Yijuan Xie ${ }^{1)}$ and Fang Wang ${ }^{2)}$ \\ 1) Department of Endocrinology, The Third Affiliated Hospital of Guangzhou Medical University, Guangzhou 510150, China
2) Department of Obstetrics and Gynecology, The Third Affiliated Hospital of Guangzhou Medical University, Guangzhou 510150,
China
}

\begin{abstract}
The study was to investigate circulating zinc- $\alpha 2$-glycoprotein $(\mathrm{ZAG})$ concentrations in women with PCOS, and changes in ZAG levels after exenatide or metformin treatment. One hundred eighty-two women with polycystic ovary syndrome (PCOS) who met the 2003 Rotterdam diagnostic criteria and 150 controls without PCOS were recruited. We partitioned women with PCOS into groups according to body mass index or blood glucose concentrations, determined serum ZAG, anthropometric parameters, metabolic and endocrine indicators, and inflammatory markers, and statistically analyzed the results. Eighty-two overweight/obese subjects of the recruited women with PCOS were then randomly assigned to groups administered either 12 weeks of exenatide injection $(10 \mu \mathrm{g}$ b.i.d. $)$ or oral metformin (1,000 $\mathrm{mg}$ b.i.d.). Circulating ZAG levels were determined after 12 weeks of treatment. The results showed that circulating ZAG was significantly lower in PCOS women than in healthy women $(p<0.01)$. Overweight/obese women and those with higher blood glucose levels had lower circulating ZAG. After 12 weeks of exenatide or metformin treatment, there were significant increases $(p<0.01)$ in circulating ZAG in both treatment groups (the exenatide baseline level was $46.54 \pm 2.38 \mathrm{ng} / \mathrm{mL} v s .56 .41 \pm 2.02 \mathrm{ng} / \mathrm{mL}$ after treatment, $p<0.01$; metformin baseline was $47.81 \pm 2.14 \mathrm{ng} / \mathrm{mL} v s .55 .67 \pm 2.01 \mathrm{ng} / \mathrm{mL}$ after treatment, $p<0.01$ ), however there was no statistical difference between the 2 treatments $(p>0.05)$. Circulating ZAG is closely related to PCOS and could be an important adipokine involved in the occurrence and development of PCOS. ZAG might possibly be applicable as a new observational indicator in the treatment of PCOS.
\end{abstract}

Key words: Zinc- $\alpha 2$-glycoprotein, Polycystic ovarian syndrome, Exenatide, Obesity, Insulin resistance

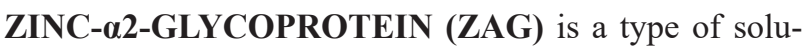
ble protein expressed in and secreted by subcutaneous and visceral adipose tissue. In recent years, circulating ZAG has been found to be associated with obesity, insulin resistance (IR), type 2 diabetes, and metabolic syndrome [1-4] and shows a clear circadian rhythm in humans [5]. Polycystic ovary syndrome (PCOS), the most common cause of menstrual disorders and anovulatory infertility in women of childbearing age, is often accompanied by obesity, IR, and hyperinsulinemia. Some studies have found that ZAG levels were significantly lower in women with PCOS compared to normal

Submitted Apr. 14, 2018; Accepted Feb. 27, 2019 as EJ18-0153

Released online in J-STAGE as advance publication Mar. 26, 2019 Correspondence to: Ying Zhang, Department of Endocrinology, The third affiliated hospital of Guangzhou Medical University, No.63, Duobao Road, Liwan District, Guangzhou, Guangdong 510150, China.

E-mail: zhangying30412@163.com

*These Authors contributed equally to the work. insulin-sensitive women $[1,5,6]$. Furthermore, Lai et al. [5] found that serum ZAG was significantly associated with PCOS and may be an IR-related cytokine in women with PCOS. Nevertheless, there are currently no reports that indicate ZAG involvement in obesity and IR in PCOS, or those that determine whether circulating ZAG is related to patient hyperandrogenism or other metabolic characteristics, or whether drug therapies for PCOS affect the level of ZAG. The purpose of this study, then, was to observe characteristics of serum ZAG and its relationship with endocrine and metabolic indicators of PCOS patients, and to explore the effects of exenatide or metformin on serum ZAG levels in overweight/obese PCOS patients.

\section{Methods}

\section{Patients}

We enrolled into our study 182 patients 18 to 40 years of age who were diagnosed with PCOS as outpatients at 
the Endocrinology Department of the Third Affiliated Hospital of Guangzhou Medical University. Diagnosis referred to the Rotterdam criteria [7]: (1) anovulation or irregular ovulation; (2) clinical or biochemical evidence of elevated androgen levels (e.g., hirsutism, acne); (3) polycystic ovarian changes (at least 12 small follicles with a diameter of $2-9 \mathrm{~mm}$ on each ovary and/or unilateral or bilateral ovarian volume $\geq 10 \mathrm{~mL}$ ). We confirmed 2 of the 3 above and excluded hyperprolactinemia, congenital adrenal hyperplasia, and other adrenal or ovarian tumors that produce testosterone. At the same time, we enrolled 150 healthy women of childbearing age as controls (18 to 40 years old).

\section{Study design and treatments}

According to the Chinese Obesity Working Group Standard [8], PCOS patients were divided into a normal weight group (NW group, 85 cases) and an overweight/ obesity group (OW/OB group, 97 cases); and adopting the 1999 WHO diabetes standards, patients were separated into a normal blood glucose group (NGT group, 102 cases), impaired blood glucose regulation group (IGR group, 62 cases), or a type 2 diabetes group (T2DM group, 18 cases). General clinical indicators, biochemical and hormonal indicators, and serum ZAG concentrations were determined and analyzed statistically.

82 of the 97 OW/OB PCOS patients were randomly and equally assigned to the exenatide (EXE group, 41 cases) or metformin group (MET group, 41 cases). 13 patients with diabetes and 2 patients who could not continue participation in drug trials for personal reasons were excluded. Both groups of patients received the same lifestyle instructions. Individuals in the EXE group were injected subcutaneously (s.c.) with exenatide (Byetta, $10 \mu \mathrm{g}$ b.i.d.) (Baxter Pharmaceutical Solutions LLC, $2.4 \mathrm{~mL} / \mathrm{vessel}$ ), and the MET group was administered metformin orally $(1,000 \mathrm{mg}$ b.i.d.) (US-Shanghai Squibb Pharmaceuticals Co., Ltd., $500 \mathrm{mg} /$ tablet). ZAG levels were determined at pre-treatment baseline and in week 12. This study was subject to approval by the Ethics Committee of the Third Affiliated Hospital of Guangzhou Medical University, and we obtained informed consent from all subjects. This prospective study has been submitted to the World Health Organization (WHO)China Clinical Trials Registry for approval and registration number designation (ChiCTR-IIR-16008084).

\section{Indicators collected}

Body weight, height, abdominal girth (AG), and hip girth were measured without shoes or outer clothing, and body mass index (BMI) and waist-to-hip ratio (WHR) were calculated. Subjects were fasted for 8 to 10 hours overnight and we collected fasting venous blood to deter- mine fasting blood glucose, fasting insulin, lipids (total cholesterol [TC], triglycerides [TG], high-density lipoprotein cholesterol [HDL-C], low-density lipoprotein cholesterol [LDL-C]), hypersensitive C-reactive protein (hs-CRP) and ZAG. Afterward, an OGTT was performed $(75 \mathrm{~g})$ and at 1,2 , and $3 \mathrm{~h}$ venous blood was taken to determine blood glucose and insulin concentrations. We then generated the following calculations. The homeostasis model assessment of insulin resistance (HOMA-IR) $=$ fasting blood glucose $(\mathrm{FBG})(\mathrm{mmol} / \mathrm{L}) \times$ fasting insulin $($ FINS) $(\mathrm{mU} / \mathrm{L}) / 22.5$. The insulin sensitivity index $($ ISI $)=$ $1 /(\mathrm{FINS}(\mathrm{mU} / \mathrm{L}) \times \mathrm{FBG}(\mathrm{mmol} / \mathrm{L}))$. The approximate trapezoidal method was performed to estimate the area under the blood glucose curve $\left(\mathrm{G}_{\mathrm{AUC}}\right)[\mathrm{mmol} / \mathrm{L} \times \mathrm{h}]$ and the area under the insulin curve $\left(\mathrm{INS}_{\mathrm{AUC}}\right)[\mathrm{mU} / \mathrm{L} \times \mathrm{h}]$. $\mathrm{G}_{\mathrm{AUC}}[\mathrm{mmol} / \mathrm{L} \times \mathrm{h}]=((\mathrm{FBG}[\mathrm{mmol} / \mathrm{L}]+3$-h postglucose load blood glucose (3hPBG) $[\mathrm{mmol} / \mathrm{L}]) / 2+1$-h postglucose load blood glucose (1hPBG) [mmol/L] + 2-h postglucose load plasma glucose $(2 \mathrm{hPBG})[\mathrm{mmol} / \mathrm{L}]) \times 1[\mathrm{~h}]$. $\mathrm{INS}_{\mathrm{AUC}}[\mathrm{mU} / \mathrm{L} \times \mathrm{h}]=(($ FINS [mU/L] $)+3$-h postglucose load blood insulin (3hINS) [mU/L])/2 +1 -h postglucose load blood insulin (1hINS) [mU/L] $)+2$-h postglucose load blood insulin $(2 \mathrm{hINS})[\mathrm{mU} / \mathrm{L}]) \times 1[\mathrm{~h}]$. Venous blood samples were taken on days $3-5$ of the menstrual cycle (non-delimited date if amenorrheic) to determine total testosterone (T), luteinizing hormone (LH), folliclestimulating hormone (FSH), and dehydroepiandronate sulfate (DHEAS) and sex-hormone binding globulin (SHBG). LH/FSH and the free androgen index $(\mathrm{FAI}=\mathrm{T}$ $[\mathrm{nmol} / \mathrm{L}] \times 100 / \mathrm{SHBG}[\mathrm{nmol} / \mathrm{L}])$ were calculated. Automatic biochemical analyses were performed for blood glucose, lipid profile, and hs-CRP. We used chemiluminescence to detect insulin and sex hormones. Circulating ZAG levels were determined by enzyme-linked immunosorbent assay (ELISA, Ray Biotech, product number: EIA-ZAG, USA), with a detection sensitivity of 0.21 $\mu \mathrm{g} / \mathrm{mL}$, and intra-assay and inter-assay coefficients of variation of $<4.7 \%$ and $<6.6 \%$, respectively.

\section{Statistical analyses}

SPSS19.0 software was used for statistical analysis, and the data was expressed as means \pm SEM. $T$-tests and analysis of variance were used for comparison between/ among groups. The correlations between ZAG and other indicators were analyzed using Pearson correlation analysis and multiple linear regression analysis. Patients in the obese group who met the appropriate conditions were randomized to exenatide or metformin groups through randomizing software (Microsoft Office professional Plus 2013, Microsoft Corporation, USA). A repeated one-way analysis of variance was utilized to compare the changes in ZAG among treatment groups. $P$ $<0.05$ was considered to be statistically significant. 
A

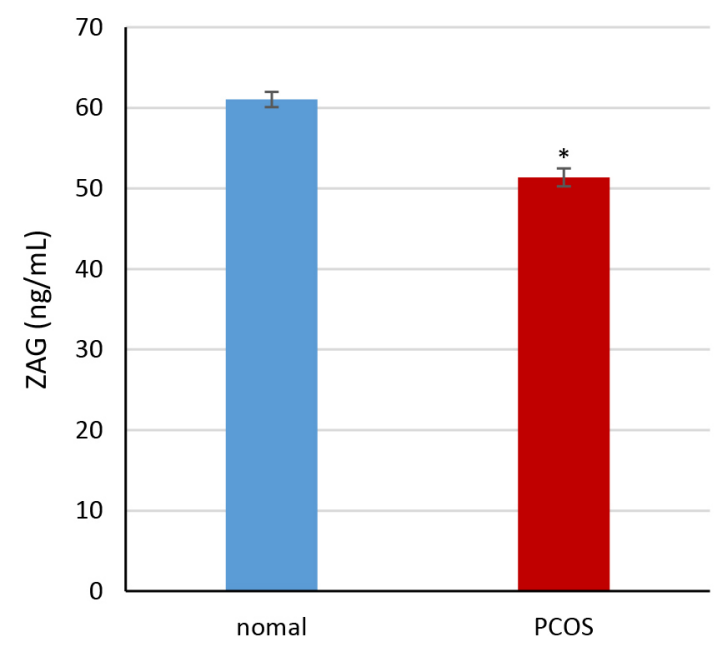

C

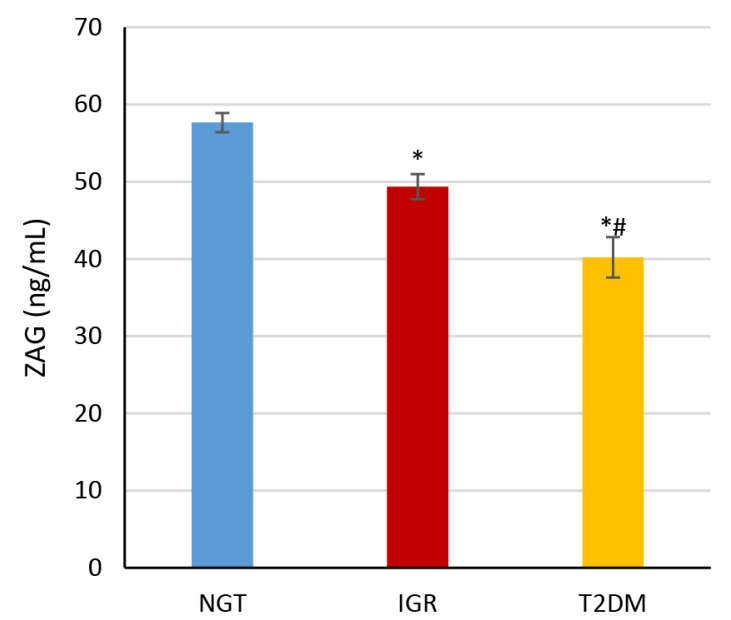

B

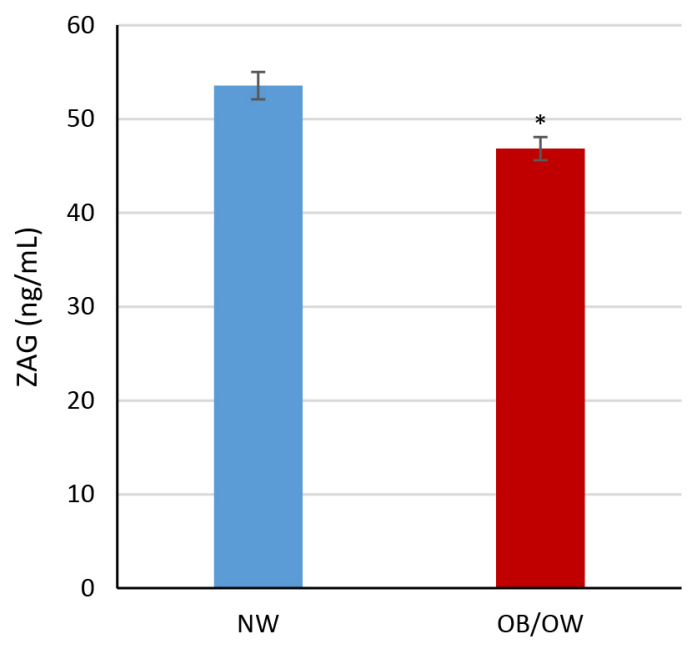

D

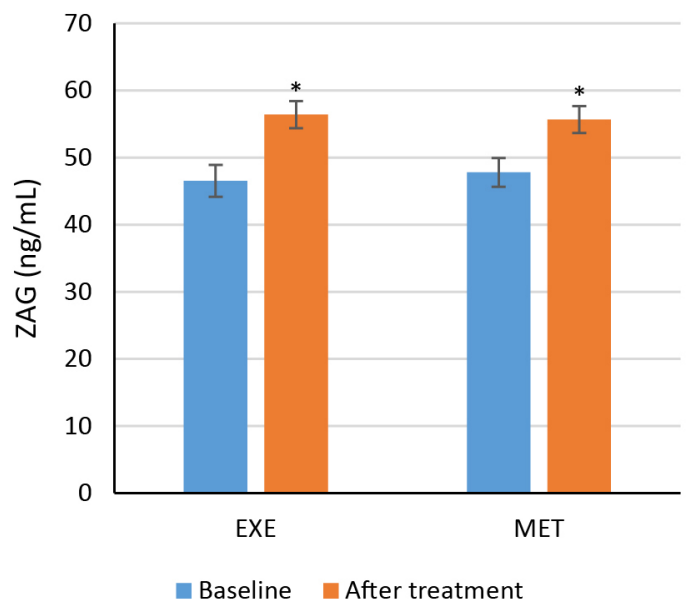

Fig. 1 A: Comparison of serum ZAG levels between PCOS and normal groups $(51.37 \pm 1.11 \mathrm{ng} / \mathrm{mL}, n=182$ vs. $61.04 \pm 0.94 \mathrm{ng} / \mathrm{mL}, n=$ 150) (*vs. normal group, $p<0.05$ ). B: Comparison of ZAG levels in PCOS patients between OW/OB and NW groups (46.86 $\pm 1.23 \mathrm{ng} / \mathrm{mL}, n=97$ vs. $53.57 \pm 1.45 \mathrm{ng} / \mathrm{mL}, n=85)(* v s$. NW group, $p<0.05)$. C: Comparison of ZAG levels in PCOS patients with T2DM $(40.21 \pm 2.63 \mu \mathrm{g} / \mathrm{mL}, n=18)$, IGR $(49.37 \pm 1.61 \mu \mathrm{g} / \mathrm{mL}, n=62)$, or NGT $(57.67 \pm 1.23 \mu \mathrm{g} / \mathrm{mL}, n=102)(* v s$. NGT group, $p<0.05$; \# vs. IGR group, $p<0.05$ ). D: Comparison of ZAG levels before and after treatment between patients in the EXE group $(n=31)$ and the MET group $(n=32)$ (the exenatide baseline level was $46.54 \pm 2.38 \mathrm{ng} / \mathrm{mL} v s .56 .41 \pm 2.02 \mathrm{ng} / \mathrm{mL}$ after treatment, $p<0.01$; metformin baseline was $47.81 \pm 2.14 \mathrm{ng} / \mathrm{mL} v s .55 .67 \pm 2.01 \mathrm{ng} / \mathrm{mL}$ after treatment) $(* v s$. before treatment, $p$ $<0.05)$.

\section{Results}

\section{Comparison of serum ZAG levels among groups}

The circulating ZAG level was lower in the PCOS group compared to the normal controls $(51.37 \pm 1.11$ $\mathrm{ng} / \mathrm{mL}$ vs. $61.04 \pm 0.94 \mathrm{ng} / \mathrm{mL}, p<0.05$ ) (Fig. $1 \mathrm{~A})$. ZAG was also lower in the $\mathrm{OW} / \mathrm{OB}$ group relative to the $\mathrm{NW}$ group $(46.86 \pm 1.23 \mathrm{ng} / \mathrm{mL}$ vs. $53.57 \pm 1.45 \mathrm{ng} / \mathrm{mL}, p<$ 0.05 ) (Fig. 1B). Analysis of variance showed that there was a statistically significant difference in serum ZAG levels among NGT, IGR, and T2DM groups $(p<0.05)$. Serum ZAG levels in the T2DM group $(40.21 \pm 2.63$ $\mu \mathrm{g} / \mathrm{mL})$ were lower than those in IGR group (49.37 \pm 1.61 $\mu \mathrm{g} / \mathrm{mL})$ and the NGT group $(57.67 \pm 1.23 \mu \mathrm{g} / \mathrm{mL})$; and serum ZAG levels in the IGR group were lower than those in the NGT group $(p<0.05)$ (Fig. 1C).

\section{Correlation between serum ZAG level and clinical variables}

Pearson correlation analysis showed that serum ZAG was negatively correlated with BMI, WHR, AG, TG, TC, FBG, FINS, 2hINS, HOMA-IR, G ${ }_{\mathrm{AUC}}$, FAI, and hsCRP; but had a positive correlation with HDL-C, ISI and SHBG. After correction for age, the above correlations 
Table 1 Correlation between serum ZAG levels and clinical variables, and multiple stepwise regression analysis

\begin{tabular}{|c|c|c|c|c|c|c|}
\hline \multirow{2}{*}{ Variable } & \multicolumn{2}{|c|}{ Pearson correlation analysis } & \multicolumn{2}{|c|}{ Pearson correlation analysis (age adjusted) } & \multicolumn{2}{|c|}{ Multiple linear regression analysis } \\
\hline & $r$ & $p$ & $r$ & $p$ & $\beta$ & $p$ \\
\hline Age (yr) & 0.173 & 0.012 & - & - & - & - \\
\hline Weight (kg) & -0.366 & $<0.001$ & -0.341 & $<0.001$ & - & - \\
\hline BMI $\left(\mathrm{kg} / \mathrm{m}^{2}\right)$ & -0.412 & $<0.001$ & -0.422 & $<0.001$ & -1.427 & 0.003 \\
\hline $\mathrm{AG}(\mathrm{cm})$ & -0.335 & $<0.001$ & -0.327 & $<0.001$ & - & - \\
\hline WHR & -0.198 & 0.008 & -0.203 & 0.007 & - & - \\
\hline TG (mmol/L) & -0.329 & $<0.001$ & -0.313 & $<0.001$ & - & - \\
\hline $\mathrm{TC}(\mathrm{mmol} / \mathrm{L})$ & -0.214 & 0.004 & -0.225 & 0.003 & - & - \\
\hline HDL-C (mmol/L) & 0.226 & 0.002 & 0.211 & 0.004 & - & - \\
\hline LDL-C (mmol/L) & -0.037 & 0.654 & -0.045 & 0.613 & - & - \\
\hline FBG $(\mathrm{mmol} / \mathrm{L})$ & -0.181 & 0.008 & -0.173 & 0.012 & - & - \\
\hline 2hPBG (mmol/L) & -0.022 & 0.679 & -0.021 & 0.682 & - & - \\
\hline FINS (mU/L) & -0.231 & 0.002 & -0.236 & 0.005 & - & - \\
\hline 2hINS (mU/L) & -0.254 & $<0.001$ & -0.259 & $<0.001$ & - & - \\
\hline HOMA-IR & -0.261 & $<0.001$ & -0.268 & $<0.001$ & - & - \\
\hline ISI & 0.378 & $<0.001$ & 0.398 & $<0.001$ & - & - \\
\hline $\mathrm{G}_{\mathrm{AUC}}(\mathrm{mmol} / \mathrm{L} \times \mathrm{h})$ & -0.235 & $<0.001$ & -0.236 & $<0.001$ & - & - \\
\hline $\mathrm{INS}_{\mathrm{AUC}}(\mathrm{mU} / \mathrm{L} \times \mathrm{h})$ & -0.279 & $<0.001$ & -0.304 & $<0.001$ & - & - \\
\hline $\mathrm{T}(\mathrm{nmol} / \mathrm{L})$ & -0.034 & 0.662 & -0.041 & 0.598 & - & - \\
\hline FAI & -0.188 & 0.01 & -0.194 & 0.01 & - & - \\
\hline LH (U/L) & 0.103 & 0.131 & 0.1 & 0.145 & - & - \\
\hline LH/FSH & 0.043 & 0.56 & 0.041 & 0.579 & - & - \\
\hline DHEAS ( $\mu \mathrm{g} / \mathrm{dL})$ & -0.016 & 0.799 & -0.031 & 0.631 & - & - \\
\hline SHBG (nmol/L) & 0.218 & 0.004 & 0.216 & 0.004 & - & - \\
\hline hs-CRP (mg/L) & -0.287 & $<0.001$ & -0.304 & $<0.001$ & - & - \\
\hline
\end{tabular}

BMI, body mass index; AG, abdominal girth; WHR, waist-to-hip ratio; TG, total cholesterol; TC, triglycerides; HDL-C, high-density lipoprotein cholesterol; LDL-C, low-density lipoprotein cholesterol; FBG, fasting blood glucose; 2hPBG, 2-hours postglucose load plasma glucose; FINS, fasting insulin; 2hINS, 2-hours postglucose load blood insulin; HOMA-IR, the homeostasis model assessment of insulin resistance; ISI, the insulin sensitivity index; $\mathrm{G}_{\mathrm{AUC}}$, the area under the blood glucose curve; $\mathrm{INS}_{\mathrm{AUC}}$, the area under the insulin curve; $\mathrm{T}$, total testosterone; DHEAS, dehydroepiandronate sulfate; SHBG, sex-hormone binding globulin; LH, luteinizing hormone; hs-CRP, hypersensitive C-reactive protein; FAI, the free androgen index; LH/FSH, luteinizing hormone/follicle-stimulating hormone. In multivariate stepwise regression analysis analysis, values included for analysis were BMI, WHR, AG, TG, TC, HDL-C, FINS, 2hINS, HOMA-IR, ISI, INS $\mathrm{AUC}_{\mathrm{AC}}$ and SHBG.

were still statistically significant $(p<0.05)$. However, there was no significant correlation between serum ZAG and LDL-C, 2hPBG, T, LH, LH/FSH, or DHEAS (Table 1). Multivariate stepwise regression analysis was performed on PCOS patients with serum ZAG as the dependent variable and BMI, WHR, AG, TG, TC, HDLC, FINS, 2hINS, HOMA-IR, ISI, INS AUC $_{\text {and }}$ SHBG as the independent variables; and the results showed that BMI was an independent factor for serum ZAG ( $\beta=$ $-1.427, t=-1.276, p=0.003)($ Table 1$)$.

\section{Effects of exenatide and metformin on serum ZAG level and clinical variables}

Changes in serum ZAG levels before and after treatment are shown in Fig. 1D and Table 2. After 12 weeks of exenatide or metformin treatment, we observed a significant increase in circulating ZAG in both treatment groups (EXE baseline, $46.54 \pm 2.38 \mathrm{ng} / \mathrm{mL}$ vs. 56.41 $\pm 2.02 \mathrm{ng} / \mathrm{mL}$ after treatment; $p<0.01$; metformin baseline, $47.81 \pm 2.14 \mathrm{ng} / \mathrm{mL}$ vs. $55.67 \pm 2.01 \mathrm{ng} / \mathrm{mL}$ after treatment; $p<0.01$ ); but there was no significant difference in the change of serum ZAG level between the 2 
Table 2 Effects of exenatide or metformin treatment on serum ZAG levels and clinical variables in overweight/obese PCOS women

\begin{tabular}{|c|c|c|c|c|c|}
\hline \multirow{2}{*}{ Variable } & \multicolumn{2}{|c|}{ EXE group $(n=31)$} & \multicolumn{2}{|c|}{ MET group $(n=32)$} & \multirow{2}{*}{$p$ value } \\
\hline & Baseline & After treatment & Baseline & After treatment & \\
\hline Age (yr) & $27.2 \pm 1.76$ & - & $27.7 \pm 1.64$ & - & \\
\hline ZAG (ng/mL) & $46.54 \pm 2.38$ & $56.41 \pm 2.02$ & $47.81 \pm 2.14$ & $55.67 \pm 2.01$ & $\mathrm{~T}=0.033$ \\
\hline Weight (kg) & $71.84 \pm 3.7$ & $66.64 \pm 3.76$ & $72.04 \pm 3.49$ & $68.49 \pm 3.50$ & $\mathrm{~T}<0.001 ; \mathrm{I}=0.009$ \\
\hline BMI $\left(\mathrm{kg} / \mathrm{m}^{2}\right)$ & $28.27 \pm 2.2$ & $26.12 \pm 2.28$ & $28.66 \pm 2.15$ & $27.27 \pm 2.03$ & $\mathrm{~T}=0.008 ; \mathrm{I}=0.024$ \\
\hline $\mathrm{AG}(\mathrm{cm})$ & $91.20 \pm 3.62$ & $85.16 \pm 3.63$ & $93.40 \pm 3.41$ & $90.52 \pm 3.30$ & $\mathrm{~T}=0.013 ; \mathrm{I}=0.017$ \\
\hline WHR & $0.89 \pm 0.28$ & $0.86 \pm 0.30$ & $0.87 \pm 0.26$ & $0.87 \pm 0.28$ & NS \\
\hline TG (mmol/L) & $1.71 \pm 0.93$ & $1.51 \pm 0.92$ & $1.68 \pm 0.92$ & $1.53 \pm 0.84$ & NS \\
\hline $\mathrm{TC}(\mathrm{mmol} / \mathrm{L})$ & $4.94 \pm 0.89$ & $4.77 \pm 0.82$ & $4.78 \pm 0.89$ & $4.49 \pm 0.86$ & NS \\
\hline HDL-C (mmol/L) & $1.26 \pm 0.62$ & $1.27 \pm 0.66$ & $1.25 \pm 0.53$ & $1.43 \pm 0.52$ & NS \\
\hline LDL-C (mmol/L) & $3.44 \pm 1.03$ & $3.42 \pm 1.05$ & $3.41 \pm 1.05$ & $3.37 \pm 1.07$ & NS \\
\hline $\mathrm{FBG}(\mathrm{mmol} / \mathrm{L})$ & $4.90 \pm 0.57$ & $4.74 \pm 0.51$ & $4.91 \pm 0.50$ & $4.76 \pm 0.41$ & NS \\
\hline 2hPBG (mmol/L) & $7.84 \pm 1.15$ & $6.21 \pm 0.46$ & $7.71 \pm 1.17$ & $5.76 \pm 1.15$ & $\mathrm{~T}<0.001$ \\
\hline FINS (mU/L) & $18.71 \pm 2.55$ & $13.12 \pm 2.24$ & $17.67 \pm 2.68$ & $13.65 \pm 2.12$ & $\mathrm{~T}=0.016 ; \mathrm{I}=0.037$ \\
\hline 2hINS (mU/L) & $146.35 \pm 5.12$ & $92.42 \pm 4.5$ & $144 \pm 5.44$ & $108.03 \pm 4.65$ & $\mathrm{~T}<0.001 ; \mathrm{I}<0.001$ \\
\hline HOMA-IR & $4.24 \pm 1.32$ & $2.68 \pm 1.09$ & $3.86 \pm 1.37$ & $2.91 \pm 1.11$ & $\mathrm{~T}<0.001 ; \mathrm{I}=0.004$ \\
\hline ISI & $0.010 \pm 0.09$ & $0.017 \pm 0.08$ & $0.012 \pm 0.08$ & $0.016 \pm 0.08$ & $\mathrm{~T}<0.001 ; \mathrm{I}=0.021$ \\
\hline $\mathrm{G}_{\mathrm{AUC}}(\mathrm{mmol} / \mathrm{L} \times \mathrm{h})$ & $23.16 \pm 2.36$ & $21.25 \pm 2.30$ & $23.93 \pm 2.37$ & $20.87 \pm 2.56$ & NS \\
\hline $\mathrm{INS}_{\mathrm{AUC}}(\mathrm{mU} / \mathrm{L} \times \mathrm{h})$ & $350.64 \pm 11.90$ & $198.78 \pm 10.65$ & $326.05 \pm 12.49$ & $233.66 \pm 12.23$ & $\mathrm{~T}<0.001 ; \mathrm{I}<0.001$ \\
\hline $\mathrm{T}(\mathrm{nmol} / \mathrm{L})$ & $1.98 \pm 0.90$ & $1.75 \pm 0.78$ & $2.03 \pm 1.10$ & $1.91 \pm 1.12$ & NS \\
\hline FAI & $10.36 \pm 2.74$ & $7.28 \pm 2.54$ & $10.86 \pm 3.26$ & $7.66 \pm 2.73$ & $\mathrm{~T}=0.022$ \\
\hline LH (U/L) & $7.55 \pm 2.57$ & $5.85 \pm 2.27$ & $6.91 \pm 1.92$ & $8.54 \pm 1.93$ & NS \\
\hline LH/FSH & $1.66 \pm 0.96$ & $1.70 \pm 0.98$ & $1.71 \pm 1.05$ & $1.71 \pm 0.99$ & NS \\
\hline DHEAS $(\mu \mathrm{g} / \mathrm{dL})$ & $263.17 \pm 10.38$ & $265.86 \pm 10.60$ & $271.04 \pm 10.50$ & $268.61 \pm 10.44$ & NS \\
\hline SHBG (nmol/L) & $31.13 \pm 4.43$ & $37.16 \pm 4.59$ & $29.59 \pm 4.63$ & $36.04 \pm 4.48$ & $\mathrm{~T}<0.001$ \\
\hline hs-CRP (mg/L) & $3.57 \pm 1.44$ & $1.61 \pm 1.21$ & $3.55 \pm 1.40$ & $1.93 \pm 0.86$ & $\mathrm{~T}<0.001 ; \mathrm{I}=0.016$ \\
\hline
\end{tabular}

$p$ value: T: Comparison between two groups, $<0.05$ is statistically significant; I: Comparison of ZAG levels before and after treatment, $<0.05$ is statistically significant; NS: No statistical significance.

treatments $(p>0.05)$.

After 12 weeks treatment, the average weight of EXE group was reduced by $3.1 \pm 1.3 \mathrm{~kg}(p<0.001)$ and MET group reduced by $1.2 \pm 0.9 \mathrm{~kg}(p<0.001)$, and EXE group was more effective than MET group in weight loss $(p=0.009)$. We observed a significant decrease in BMI $(p=0.008)$ and $\mathrm{AG}(p=0.013)$ in both treatment groups, and EXE group was better in changes of BMI and $A G$ than MET group ( $p=0.024 ; p=0.017)$. There was no statistical change in WHR in both treatments $(p>0.05)$.

There were no significant changes of FPG and $\mathrm{G}_{\mathrm{AUC}}$ in both EXE group and MET group $(p>0.05)$ after 12 weeks treatment. There was a significant reduction of $2 \mathrm{hPBG}$ in both groups, though there was no significant difference between the 2 treatments $(p>0.05)$. FINS,
2hINS, HOMA-IR and $\mathrm{INS}_{\text {AUC }}(p=0.016 ; p<0.001 ; p$ $<0.001 ; p<0.001)$ were significantly decreased, and ISI $(p<0.001)$ significantly increased after treatment in both groups. The improvements of FINS, 2hINS, HOMA-IR, $\mathrm{INS}_{\mathrm{AUC}}$ and ISI were more effective in EXE group than metformin group $(p=0.037 ; p<0.001 ; p=0.004 ; p<$ $0.001 ; p=0.021)$. There were not any significant changes of TC, TG, HDL-C and LDL-C after 12 weeks of treatment in both groups $(p=0.022 ; p<0.001)$, though there were in both treatments $(p>0.05)$. FAI was significantly reduced and SHBG increased no significant differences between the 2 treatments $(p>0.05)$. There were not any significant changes of T, LH, LH/FSH and DHEAS in both treatments $(p>0.05)$. The hs-CRP levels in the two groups were significantly reduced after 
treatment $(p<0.001)$, and EXE group was more effective than MET group in reducing hs-CRP $(p=0.016)$. Changes in clinical variables before and after treatment are all set out in Table 2.

Multivariate stepwise regression analysis was performed using change of serum ZAG as the dependent variable and changes of weight, BMI, HOMA-IR, $2 \mathrm{hPBG}, \mathrm{TC}$ and hs-CRP as independent variables; and the results shows that changes of weight and HOMA-IR were independent factor for change of serum ZAG $(\beta=$ $-0.872, t=-2.128, p=0.032 ; \beta=-1.361, t=-2.289 p=$ $0.035)$.

\section{Discussion}

Previous studies have shown that ZAG derived from adipose tissue plays a critical role in the regulation of obesity [9-11], that its concentration increases proportionally with the degree of weight loss [10], and that diminished ZAG in the adipose tissue of obese individuals reduces fat mobilization [12]. As a heterogeneous disease, PCOS often includes obesity and IR. The present study revealed that serum ZAG levels were significantly lower in PCOS patients than in normal women, and that overweight and obese patients had lower ZAG levels than normal-weight patients. These findings are consistent with the results from Lai et al. [5]. It is suggested that PCOS patients possess poorer mobility than the normal population and are more prone to obesity, which may be related to the decrease in serum ZAG levels. In animal experiments, ZAG-knockout rats were more likely to gain weight when given a high-fat diet; and there was no reaction with $\beta 3$-adrenergic receptors, producing weakened lipolysis [13]. ZAG may activate AMP kinases to regulate ATP-consuming activities (fatty acid synthesis, glycogen synthesis, and lipolysis), and ATP-production processes (glycogen breakdown and $\beta$-oxidation) [14]. In our study, serum ZAG levels were related to other indicators: they showed a significant negative correlation with obesity (WHR and BMI), insulin levels, and HOMA-IR; and a significant positive correlation with ISI. Multiple linear regression analysis suggested that BMI was an independent factor for ZAG, and that ZAG was closely related to obesity and IR in patients with PCOS. ZAG may therefore be an adipokine, which upon attenuation might be involved in the pathogenic development of PCOS in women.

In this study we first grouped the blood glucose levels of PCOS patients and found that serum ZAG levels were lower in the T2DM group relative to the IGR group and the NGT group. Serum ZAG levels were also lower in the IGR group compared to the NGT group. Correlation analysis showed that ZAG was negatively correlated with FBG and $\mathrm{G}_{\mathrm{AUC}}$, suggesting that $\mathrm{ZAG}$ is closely related to blood glucose levels in PCOS patients; this may be due to ZAG's participation in the development of the patient's IR, thus affecting the regulation of blood glucose levels. In addition, correlation analysis also found that serum ZAG levels were negatively related to serum TG and TC, but positively correlated with HDL$\mathrm{C}$; suggesting that a decrease in ZAG levels in PCOS patients leads to abnormal blood lipid levels. Previous studies have found that macrophage culture medium or tumor necrosis factor- $\alpha$ treatment of primary human adipocytes reduced expression and secretion of ZAG [15], indicating that the inflammatory state of obese individuals reduces ZAG levels of adipose tissue. In the present study hs-CRP was used as an indicator to detect the inflammatory state of the body and to predict the risk for cardiovascular disease; and we found that circulating ZAG levels were significantly correlated with hs-CRP levels, suggesting to us that ZAG participates in the inflammatory state observed generally for PCOS patients.

We herein invoked FAI as a reference index for diagnosing hyperandrogenism in PCOS patients [16]. It was noted that serum ZAG levels were significantly negatively correlated with FAI levels, suggesting that ZAG is associated with the development of hyperandrogenism. This might occur via indirect participation in the development of IR and hyperinsulinemia so as to affect androgen levels in PCOS patients. Previously, Lai et al. [5] found that PCOS patients with high FAI had higher HOMA-IR and lower ZAG. By stimulating $17 \alpha$-hydroxylase/17,20-lyase and $3 \beta$-hydroxysteroid dehydrogenase in the ovary, hyperinsulinemia directly stimulates ovarian androgen secretion, reduces SHBG levels, and inhibits the production of hepatic insulin-like growth factor binding protein-1 (IGFBP-1) [17]. These effects might also stimulate adrenal $17 \alpha$-hydroxylase/ 17, 20-lyase to increase response to ACTH [18]. Collectively, these actions could induce hyperandrogenism in PCOS patients, although the relationships among ZAG, IR, and hyperandrogenism require further study.

Glucagon-like peptide-1 receptor agonist (GLP-1RA) are distributed in many tissues and organs in the body, and play other roles besides controlling blood sugar, such as cardiovascular protection, treatment of obesity, improvement of fatty liver, etc. [19]. Furthermore, previous clinical trials and other small-sample studies found that GLP-1RA effectively reduced weight, improved IR and metabolic disorders, improved menstrual cyclicity, and increased pregnancy rate in PCOS [20-25]. This study found that metformin group and exenatide group were significantly effective in reducing body weight,

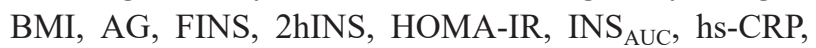
and elevating ISI. After 12 weeks of treatment, exenatide 
appears to be more effective in improving obesity symptoms, improving insulin resistance, and controlling metabolic inflammation than metformin, the classic treatment drug of PCOS. This might provide clinical evidence for exenatide as another drug option for treatment of PCOS. The present study was the first to observe changes in serum ZAG levels before and after treatment in $\mathrm{OW} / \mathrm{OB}$ PCOS patients and to explore whether serum ZAG levels would be changed while clinical, endocrine metabolic indicators being improved. We found that for Chinese PCOS patients, both treatments significantly increased serum ZAG levels and multiple linear regression analysis showed that changes of body weight and HOMA-IR were Independent impact factors of ZAG changes, suggesting that improvements in obesity and insulin resistance in drug therapy led to improvements in ZAG levels. Yang et al. [1] used liraglutide for treating diabetic patients and found that ZAG levels significantly elevated, which was considered to be related to the improvement of IR in diabetic patients. However, there was no significant difference in the changes of ZAG between the two groups in this study. It was not excluded that the treatment time was short and the difference between the two treatments could not be seen. The effects and mechanisms of exenatide and metformin treatments on serum $\mathrm{ZAG}$ require further trials.

In conclusion, our study confirmed that circulating ZAG decreased in PCOS, especially in obese women or those with high blood glucose. Associations between ZAG expression and obesity, insulin resistance, glucose and lipid metabolism, inflammatory status, and hyper- androgenism in patients might therefore be involved in the development of PCOS. Furthermore, our study also found that treatments up-regulated serum ZAG levels in PCOS patients. Circulating ZAG is closely related to PCOS and might be an important adipokine involved in the occurrence and development of PCOS. ZAG might also be used as a new observational indicator for treatment of PCOS. A larger sample size, longer longitudinal evaluation, and more intensive studies are needed to appropriately investigate the characteristics of ZAG levels in PCOS patients, and possible underlying mechanisms of action.

\section{Acknowledgments}

We thank the participating outpatients at the Endocrinology Department of the Third Affiliated Hospital of Guangzhou Medical University for their valuable contributions.

\section{Disclosure}

None of the authors have any potential conflicts of interest associated with this research.

\section{Funding}

This study was supported by the National Natural Science Foundation of China (No. 81200607) and the project of the Key Laboratory for Major Obstetric Diseases of Guangdong Higher Education Institutes (No. 2012Z05).

\section{References}

1. Yang M, Liu R, Li S, Luo Y, Zhang Y, et al. (2013) Zinc$\alpha 2$-glycoprotein is associated with insulin resistance in humans and is regulated by hyperglycemia, hyperinsulinemia, or liraglutide administration: cross-sectional and interventional studies in normal subjects, insulin-resistant subjects, and subjects with newly diagnosed diabetes. Diabetes Care 36: 1074-1082.

2. Yeung DCY, Lam KSL, Wang Y, Tso AWK, Xu A (2009) Serum zinc- $\alpha 2$-glycoprotein correlates with adiposity, triglycerides, and the key components of the metabolic syndrome in Chinese subjects. J Clin Endocrinol Metab 94: 2531-2536.

3. Balaz M, Vician M, Janakova Z, Kurdiova T, Surova M, et al. (2014) Subcutaneous adipose tissue zinc- $\alpha 2-$ glycoprotein is associated with adipose tissue and wholebody insulin sensitivity. Obesity (Silver Spring) 22: 18211829.

4. Ceperuelo-Mallafre V, Naef S, Escote X, Caubet E, Gomez JM, et al. (2009) Circulating and adipose tissue gene expression of zinc- $\alpha 2$-glycoprotein in obesity: its relationship with adipokine and lipolytic gene markers in subcutaneous and visceral fat. J Clin Endocrinol Metab 94: 5062-5069.

5. Lai Y, Chen J, Li L, Yin J, He J, et al. (2016) Circulating zinc- $\alpha 2$-glycoprotein levels and insulin resistance in polycystic ovary syndrome. Sci Rep 6: 25934.

6. Qu C, Zhou X, Yang G, Li L, Liu H, et al. (2016) The natural logarithm of zinc- $\alpha 2$-glycoprotein/HOMA-IR is a better predictor of insulin sensitivity than the product of triglycerides and glucose and the other lipid ratios. Cytokine 79: 96-102.

7. Rotterdam ESHRE/ASRM-Sponsored PCOS Consensus Workshop Group (2004) Revised 2003 consensus on diagnostic criteria and long-term health risks related to polycystic ovary syndrome. Fertil Steril 81: 19-25.

8. Chen C, Lu FC (2004) The guidelines for prevention and control of overweight and obesity in Chinese adults. Biomed Environ Sci 17: 1-36. 
9. Russell ST, Tisdale MJ (2011) Studies on the anti-obesity activity of zinc- $\alpha 2$-glycoprotein in the rat. Int $J$ Obes (Lond) 35: 658-665.

10. Mracek T, Stephens NA, Gao D, Bao Y, Ross JA, et al. (2011) Enhanced ZAG production by subcutaneous adipose tissue is linked to weight loss in gastrointestinal cancer patients. Br J Cancer 104: 441-447.

11. Bing C, Mracek T, Gao D, Trayhurn P (2010) Zinc- $\alpha 2$ glycoprotein: an adipokine modulator of body fat mass \& quest. Int J Obes (Lond) 34: 1559-1565.

12. Mracek T, Ding Q, Tzanavari T, Kos K, Pinkney J, et al. (2010) The adipokine zinc- $\alpha 2$-glycoprotein (ZAG) is downregulated with fat mass expansion in obesity. Clin Endocrinol (Oxf) 72: 334-341.

13. Rolli V, Radosavljevic M, Astier V, Macquin C, CastanLaurell I, et al. (2007) Lipolysis is altered in MHC class I zinc-a2-glycoprotein deficient mice. FEBS Lett 581: 394 400.

14. Eckardt K, Schober A, Platzbecker B, Mracek T, Bing C, et al. (2011) The adipokine zinc- $\alpha 2$-glycoprotein activates AMP kinase in human primary skeletal muscle cells. Arch Physiol Biochem 117: 88-93.

15. Gao D, Trayhurn P, Bing C (2010) Macrophage-secreted factors inhibit ZAG expression and secretion by human adipocytes. Mol Cell Endocrinol 325: 135-142.

16. Li H, Xu X, Wang X, Liao X, Li L, et al. (2016) Free androgen index and Irisin in polycystic ovary syndrome. $J$ Endocrinol Invest 39: 549-556.

17. Gambineri A, Pelusi C, Vicennati V, Pagotto U, Pasquali $\mathrm{R}$ (2002) Obesity and the polycystic ovary syndrome. Int $J$ Obes Relat Metab Disord 26: 883-896.

18. Puurunen J, Piltonen T, Jaakkola P, Ruokonen A, MorinPapunen L, et al. (2009) Adrenal androgen production capacity remains high up to menopause in women with polycystic ovary syndrome. J Clin Endocrinol Metab 94:
1973-1978.

19. Gallwitz B (2014) Extra-pancreatic effects of incretinbased therapies. Endocrine 47: 360-371.

20. Elkind-Hirsch K, Marrioneaux O, Bhushan M, Vernor D, Bhushan R (2008) Comparison of single and combined treatment with exenatide and metformin on menstrual cyclicity in overweight women with polycystic ovary syndrome. J Clin Endocrinol Metab 93: 2670-2678.

21. Jensterle M, Kravos NA, Pfeifer M, Kocjan T, Janez A (2015) A 12-week treatment with the long-acting glucagon-like peptide 1 receptor agonist liraglutide leads to significant weight loss in a subset of obese women with newly diagnosed polycystic ovary syndrome. Hormones (Athens) 14: 81-90.

22. Jensterle M, Salamun V, Kocjan T, Vrtacnik Bokal E, Janez A (2015) Short term monotherapy with GLP-1 receptor agonist liraglutide or PDE 4 inhibitor roflumilast is superior to metformin in weight loss in obese PCOS women: a pilot randomized study. J Ovarian Res 8: 32.

23. Jensterle Sever M, Kocjan T, Pfeifer M, Kravos NA, Janez A (2014) Short-term combined treatment with liraglutide and metformin leads to significant weight loss in obese women with polycystic ovary syndrome and previous poor response to metformin. Eur J Endocrinol 170: 451-459.

24. Zheng SY, Zhang Y, Long T, Lu JH, Liu X, et al. (2017) Short term monotherapy with exenatide is superior to metformin in weight loss, improving insulin resistance and inflammation in Chinese overweight/obese PCOS women. Obes Med 7: 15-20.

25. Liu X, Zhang Y, Zheng SY, Lin R, Xie YJ, et al. (2017) Efficacy of exenatide on weight loss, metabolic parameters and pregnancy in overweight/obese polycystic ovary syndrome. Clin Endocrinol (Oxf) 87: 767-774. 